\title{
Hysteresis Compensation for Smart Actuators Using Inverse Generalized Prandtl-Ishlinskii model
}

\author{
Mohammad Al Janaideh, Ying Feng, Subhash Rakheja, Chun-Yi Su, and Camille Alain Rabbath
}

\begin{abstract}
Control of smart actuators is limited due to strong hysteresis effects which affect the accuracy of these actuators in micropositioning applications. In this paper, generalized Prandtl-Ishlinskii hysteresis model and its inverse are presented to characterize and to compensate hysteresis effects in smart actuators, where a generalized symmetric play operator is adopted to form the generalized Prandtl-Ishlinskii model. The capability of the formulated model to characterize hysteresis in smart actuators is demonstrated by comparing its outputs with experimental results obtained from a piezoceramics actuator. Inverse of the generalized Prandtl-Ishlinskii model is also constructed and it can be implemented as a feedforward compensator to migrate the effects of the hysteresis in different types of smart actuators. To improve the robustness and ensure the stability of the closed-loop system, a robust adaptive control is developed considering the system dynamics. The simulation results validate the effectiveness of the proposed approach.
\end{abstract}

\section{INTRODUCTION}

Hysteresis is a nonlinear phenomenon that appears in various systems including smart actuators and materials [1]-[4][7]. Smart actuators, such as piezoceramic actuators, magnetostrictive actuators, and shape memory alloy (SMA) actuators have been widely used in micropositioning applications. However, these actuators show strong hysteresis effects in their output responses. These effects can cause inaccuracy and oscillations in the system response, and could lead to instability of the closed loop system [5]. A number of hysteresis models have been developed for characterizing the hysteresis properties of materials and actuators. The primary goal of these models is to accurately characterize the hysteresis behavior to study the effects in order to facilitate the design of controllers for compensating for the hysteresis effects [1].

The most widely cited hysteresis models are: operatorbased and differential equation-based hysteresis models. Operator-based hysteresis models include Preisach model [2], Krasnosel'skii-Pokrovskii model [4], and PrandtlIshlinskii model [3], while differential equation-based hysteresis models include Duhem model [19] and Bouc-Wen model [19]. Therein, the Prandtl-Ishlinskii hysteresis model is one of the most widely used phenomological basedoperator models in applications involving hysteresis modeling and compensation of hysteresis in smart actuators [6] [12]. The hysteresis in the Prandtl-Ishlinskii model is considered as a cumulative effect of a number of play or

${ }^{1}$ M. Al Janaideh, Y. Feng, S. Rakheja,and C.-Y. Su are with Department of Mechanical and Industrial Engineering, Concordia University, Montreal, Quebec, Canada, C. A. Rabbath is with Defence Research and Development Canada, Quebec City, Canada. stop operators, which are parameterized by single threshold, and density function [3].

Based on the above formulated hysteresis models two control strategies are generally adopted in the literature to compensate hysteresis effects in smart actuators [8]-[19]. The first scheme is to design a nonlinear controller that yields input signals that directly incorporate actuator nonlinearities [12]. The second scheme is to construct the inverse of the hysteresis model and apply it as a feedforward compensator.

For the control scheme to construct the inverse of the hysteresis model, the inversion of Preisach, Krasnosel'skiiPokrovskii model, and PrandtlIshlinskii model have been addressed to compensate hysteresis effects of smart actuators e.g. [6], [10]. In [6], the hysteresis effects of a piezoceramic actuator is compensated by constructing the inverse of Prandtl-Ishlinskii model. Approximation inversion of Krasnosel'skii-Pokrovskii model was developed in [10] to mitigate the effects of hysteresis in piezoceramic actuator. For theoretical point of view, the system stability and the dynamics of the actuators should be considered. Some closed-loop control approaches are addressed based on the inverse of hysteresis model to achieve better tracking performance. In [18], inverse Preisach model is used as a feedforward compensator with PID feedback control system to reduce hysteresis effects in a piezoceramic actuator. In [21], a robust control system to compensate hysteresis effects in a magnetostrictive actuator is proposed, which is based on the inverse of Preisach model. In [15], a robust control designs for smart material actuators operating in nonlinear and hysteretic regimes is developed to achieve high tracking performance, and approximate inversion for the formulated hysteresis model is employed in a closed-loop control system.

By re-defining the play operator, the Prandtl-Ishlinskii model is extended to describe more general hysteresis shapes. In this paper, and as a further development, a corresponding inverse is also provided for compensations of the generalized Prandtl-Ishlinskii model for different smart actuators. Such compensations are illustrated by the example for the hysteresis in a piezoceramic actuator. Considering the dynamics of the actuators, the corresponding adaptive robust control law is designed to improve the system performance.

The paper is organized as follows: Section 2 describes the characteristics of the Prandtl-Ishlinskii model. Section 3 states the generalized Prandtl-Ishlinskii model based on the generalized play operator. In Section 4, the inverse of the generalized Prandtl-Ishlinskii model is presented and the simulation results for generalized Prandtl-Ishlinkii model and 
its inverse is introduced in Section 5. Consider the linear dynamics in piezoceramic actuators, a robust adaptive control is proposed and simulation results are presented in Section 6 to demonstrate the effectiveness of the approach. Finally, Section 7 concludes the paper.

\section{PRANDTL-ISHLINSKII MODEL}

In this paper, the hysteresis nonlinearity is presented by the Prandtl-Ishlinskii model. We shall introduce an essential well known hysteresis operator in order to present the generalized Prandtl-Ishlinskii model.

\section{A. Play hysteresis operator}

Play hysteresis operator is a continuous and rateindependent hysteresis operator. A detailed discussion about this operator can be found in [3]. Analytically, let $C_{m}\left[0, t_{E}\right]$ represent the space of piecewise monotone continuous functions. For any input $v(t) \in C_{m}\left[0, t_{E}\right]$, let $0=t_{0}<t_{1}<$ $\ldots,<t_{N}=t_{E}$ be a partition of $\left[0, t_{E}\right]$ such that the function $v$ is monotone on each of the sub-intervals $\left[t_{i}, t_{i+1}\right]$. Then, the output of the play operator is defined by [3]:

$$
\begin{aligned}
F_{r}[v](0)= & f_{r}(v(0), 0)=w(0) \\
w(t)= & F_{r}[v](t)=f_{r}\left(v(t), F_{r}[v]\left(t_{i}\right)\right) \\
& \text { for } t_{i}<t \leq t_{i+1} \text { and } 0 \leq i \leq N-1
\end{aligned}
$$

where $f_{r}(v, w)=\max (v-r, \min (v+r, w))$

The argument of the operator is written in square brackets to indicate the functional dependence, since it maps a function to another function. The play operator is characterized by input $v$ and the threshold $r$. Due to the nature of the play operator, the above definition is based on $v(t) \in C_{m}\left[0, t_{E}\right]$ of continuous and piecewise monotone functions. This, however, can be extended to space $C\left[0, t_{E}\right]$ of continuous functions [3].

\section{B. Prandtl-Ishlinskii Model}

The Prandtl-Ishlinskii model utilizes the play operator $F_{r}[v]$ to describe relationship between output $H[v](t)$ and input $v(t)[3]$ [6]:

$$
H[v](t)=q v(t)+\int_{0}^{R} p(r) F_{r}[v](t) d r
$$

where $p(r)$ is a density function, satisfying $p(r) \geq 0$, which is generally identified from experimental data. $q$ is a positive constant. The Prandtl-Ishlinskii model with the density function maps $C\left[t_{o}, \infty\right)$ into $C\left[t_{o}, \infty\right)$. Since the density function $p(r)$ vanishes for large values of $r$, the choice of $R=\infty$ as upper limit of integration, widely used in the literature, is just a matter of convenience [3]. The above model was applied to predict and to reduce hysteresis effects of piezoceramic actuators [6]. The reasonably good validity of the model was further demonstrated by comparing the model predictions with the measured data of a piezoceramic actuator.

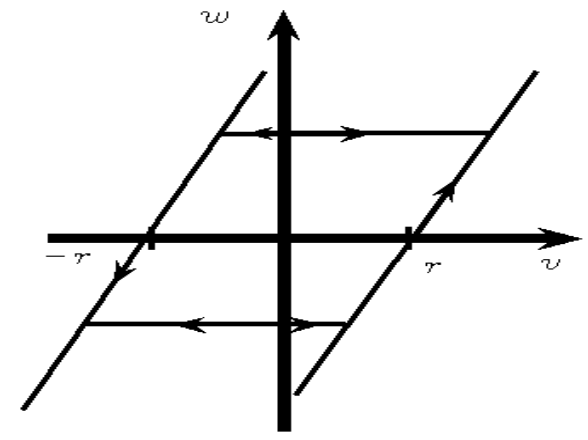

Fig. 1. Play hysteresis operator.

\section{A Generalized PRANDTL-ISHLINSKII MODEL BASED Generalized Play OPERATOR}

Owing to the nature of the play operator, the PrandtlIshlinskii model predictions were limited to symmetric hysteresis loops of piezoceramic actuators. Moreover, this model cannot show saturation property in hysteresis loops. This property has been widely demonstrated in magnetorestrictive and SMA actuators. Alternatively, the generalized play operator, described in Fig. 2, could be utilized to realize input-output relationships of the smart actuators.

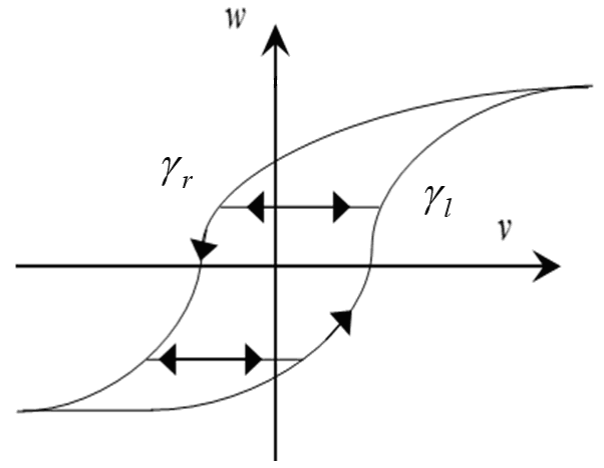

Fig. 2. Input-output relationship of a generalized play operator.

The generalized play operator is a nonlinear play operator, where an increase in input $v$ causes the output $w$ to increase along the curve $\gamma_{l}$ or a decrease in input $v$ causes the output $w$ to decrease along the curve $\gamma_{r}$, resulting in asymmetric hysteresis loops about the input or the output. The curves $\gamma_{l}$ and $\gamma_{r}$ are continuous non decreasing functions with $\gamma_{l} \leq \gamma_{r}$ [4]. Analytically, the output of the generalized play operator for any input $v(t) \in C_{m}\left[0, t_{E}\right]$ is defined by [3] [19]:

$$
\begin{aligned}
F_{l r}^{\gamma}[v](0)= & f_{l r}^{\gamma}(v(0), 0)=w(0) \\
w(t)= & F_{l r}^{\gamma}[v](t)=f_{l r}^{\gamma}\left(v(t), F_{l r}^{\gamma}[v]\left(t_{i}\right)\right) ; \\
& \text { for } t_{i}<t \leq t_{i+1} \text { and } 0 \leq i \leq N-1
\end{aligned}
$$

where $f_{l r}^{\gamma}(v, w)=\max \left(\gamma_{l}(v)-r, \min \left(\gamma_{r}(v)+r, w\right)\right)$

\section{A. Symmetric generalized play operator}

In this paper, a generalized Prandtl-Ishlinskii model is formulated using symmetric generalized play operator. Ana- 
lytically, the output of the operator is expressed as:

$$
\begin{aligned}
F_{r}^{\gamma}[v](0)= & f_{r}^{\gamma}(v(0), 0)=w(0) \\
w(t)= & F_{r}^{\gamma}[v](t)=f_{r}^{\gamma}\left(v(t), F_{r}^{\gamma}[v]\left(t_{i}\right)\right) \\
& \text { for } t_{i}<t \leq t_{i+1} \text { and } 0 \leq i \leq N-1 \\
f_{r}^{\gamma}(v, w)= & \max (\gamma(v)-r, \min (\gamma(v)+r, w))
\end{aligned}
$$

where $\gamma: R \rightarrow R$ is an envelope function; strictly increasing, continuous and odd.

Some of mathematical properties of the generalized play operator are:

- Lipschitz-continuity:

For a given input $v(t)$, Lipschitz-continuity of the generalized play operator can be ensured if the function $\gamma(v(t))$ is Lipschitz continuous [3].

- Rate-Independent:

The generalized play operator $F_{r}^{\gamma}[v]$ is rate independent hysteresis operator if:

$$
F_{r}^{\gamma}[v] o \varphi=F_{r}^{\gamma}[v o \varphi]
$$

where $\varphi$ is continuous increasing function $\varphi:[0, T]$ satisfying $\varphi(0)=0$ and $\varphi(T)=T$.

- Range :

For a given input $v(t) \in C[0, T]$ and $r \geq 0$ the range of the output of the generalized play operator is:

$$
\begin{aligned}
& \max _{t \in[0, T]} F_{r}^{\gamma}[v](t)=f_{r}\left(\max _{t \in[0, T]} \gamma(v(t)), w(0)\right) \\
& \min _{t \in[0, T]} F_{r}^{\gamma}[v](t)=f_{r}\left(\min _{t \in[0, T]} \gamma(v(t)), w(0)\right)
\end{aligned}
$$

\section{B. Generalized Prandtl-Ishlinskii model}

Generalized Prandtl-Ishlinskii model is formulated using symmetric generalized play operator $F_{r}^{\gamma}[v](t)$ to yield output $P[v](t)$ as [22]:

$$
P[v](t)=q \gamma(v(t))+\int_{0}^{R} p(r) F_{r}^{\gamma}[v](t) d r
$$

Using the generalized play operator, the generalized PrandtlIshlinskii model can be employed to characterize hysteresis effects of different smart actuators. The above analytical inverse can also be numerically implemented. The generalized model can be also described as:

$$
P(t)=q \gamma(v(t))+\sum_{j=1}^{n} p_{j} F_{r_{j}}^{\gamma}[v](t)
$$

where $n$ is the number of the generalized play operator.

Remark 1: The classical Prandtl-Ishlinskii model is a special case of the generalized model. For identical envelope function, the generalized model reduces to the classical Prandtl-Ishlinskii model, described in (2).

\section{InVERSE Generalized PRANDTL-IshLinKsil MODEL}

In this paper, inversion of the generalized PrandtlIshlinskii model is presented for the purpose of reducing the hysteresis effects in control systems. The inverse of generalized Prandtl-Ishlinskii model is used as a feedforward compensator to compensate hysteresis effects. It should be mentioned that this inverse is computed analytically. In other words, exact inverse of this model is reachable, consequently making it more attractive for real-time applications of smart actuators.

For the generalized Prandtl-Ishlinskii model in equation (9), and the generalized play operator used in equation (4), if the inverse of the envelope function $\gamma^{-1}: R \rightarrow R$ exists; the inverse of the generalized Prandtl-Ishlinskii model that is presented in (9) can be analytically expressed as:

$$
P^{-1}=\gamma^{-1}\left(H^{-1}\right)
$$

where

$$
H^{-1}[v](t)=q^{-1} v(t)+\sum_{j=1}^{n} g\left(\hat{r}_{j}\right) F_{\hat{r}_{j}}[v](t)
$$

where $\hat{r}$ is the threshold of the inverse generalized PrandtlIshlinskii model and $g$ is the density function of the inverse model.The parameters of the inverse are expressed as [6]:

$$
\begin{gathered}
q^{-1}=\frac{1}{q} \\
\hat{r}_{j}=q r_{j}+\sum_{i=1}^{j-1} p_{i}\left(r_{j-} r_{i}\right) \\
g_{j}=-\frac{p_{j}}{\left(q+\sum_{i=1}^{j} p_{i}\right)\left(q+\sum_{i=1}^{j-1} p_{i}\right)}
\end{gathered}
$$

The above analytical inverse can also be numerically implemented. Using the discrete input $v(k)$ corresponding to an interval $k(k=0,1,2,3, \ldots, N)$ as:

$$
P(k)=q \gamma(v(k))+\sum_{j=1}^{n} p_{j} F_{r_{j}}^{\gamma}[v](k)
$$

where $n$ is the number of the generalized play operators. The output of the inverse is expressed numerically as:

$$
P^{-1}(k)=\gamma^{-1}\left(q^{-1} v(k)+\sum_{j=1}^{n} g_{j} F_{\hat{r}_{j}}[v](k)\right)
$$

\section{Simulation Results For Generalized \\ PRANDTL-ISHLINKSII MODEL AND ITS INVERSE}

In this section, an input signal of the form: $v(t)=$ $3 \sin (t)+2 \cos (1.3 t)$ is considered to evaluate minor as well as major hysteresis loops. For the generalized PrandtlIshlinskii model (8), the density function is selected as $p(r)=0.1 e^{-0.1 r}, r \in[0,5]$, where the threshold $r$ is selected as:

$$
r_{j}=0.24 j j=1,2,3, \ldots, n=20
$$


and the following envelope function is used to construct the generalized Prandtl-Ishlinskii model:

$$
\gamma(v)=1.3 v-0.4
$$

The chosen simulation parameters are: $T=25, \Delta t=0.001$, $q=0.18$.

Fig.3 shows the simulation results of the generalized Prandtl-Ishlinskii model and its inverse. The hysteresis shape is shown in Fig. 3(a) based on the generalized PrandtlIshlinskii model in (8), and the corresponding inverse of the generalized Prandtl-Ishlinskii model defined in (10) is shown in Fig. 3(b), then, the compensation result is shown in Fig. 3(c). The results show the capability of the model and its inverse to compensate hysteresis effects .

Remark 2: The output of the generalized Prandtl-Ishlinskii model strongly depends upon the generalized play operator, the envelope function, and the density function. For example, the envelope function can be selected as $\gamma(v)=$ $a_{0} \tanh \left(a_{1} v+a_{2}\right)+a_{3}$ to describe the saturated hysteresis nonlinearities existing in some smart actuators, such as shape memory alloy (SMA) actuators and magnetostrictive actuators, where $a_{0}, a_{1}, a_{2}$ and $a_{3}$ are adjusted parameters.

\section{AdAPTIVE ROBUST CONTROL DESIGN}

\section{A. controller design}

For these smart material-based actuators, the construction of the inverse of the generalized Prandtl-Ishlinskii model can mitigate the effects of the unknown hysteresis nonlinearities. However, it is a open-loop approach, and does not consider the influence of the dynamics, which can degrade the system performance in high-frequency operation. Therefore, a robust adaptive controller is proposed in this section to ensure the closed-loop system stability and improve the tracking precision. As an example, the generalized Prandtl-Ishlinskii model is used to describe the hysteresis existing in the piezoceramic actuators in this section, and the linear dynamics of piezoceramic actuators is identified as a second-order linear dynamics, then the system equations of motion can be described as

$$
\ddot{x}(t)+2 \xi \omega_{n} \dot{x}(t)+\omega_{n}^{2} x(t)=z(t)
$$

where $x(t)$ is the system output (actuator displacement), $z(t)$ is the hysteretic relation between the input voltage and the excitation force. Based on the definition of generalized Prandtl-Ishlinskii model in (7), we have

$$
z(t)=P[u](t)
$$

where $u(t)$ it the input voltage.

According to the compensation scheme proposed in this paper, the output of the inverse generalized Prandtl-Ishlinskii model $P^{-1}[u](t)$ is added to mitigate the effect of hysteresis, we can obtain

$$
z(t)=P[v](t)
$$

where

$$
v(t)=P^{-1}[u](t)
$$

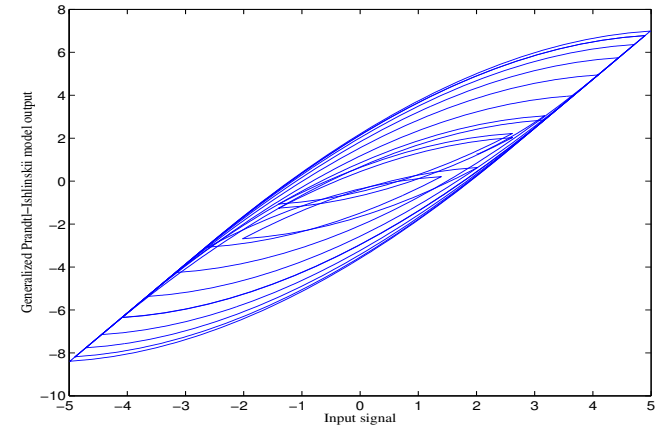

(a) Input-output relationship of the generalized Prandtl-Ishlinskii model

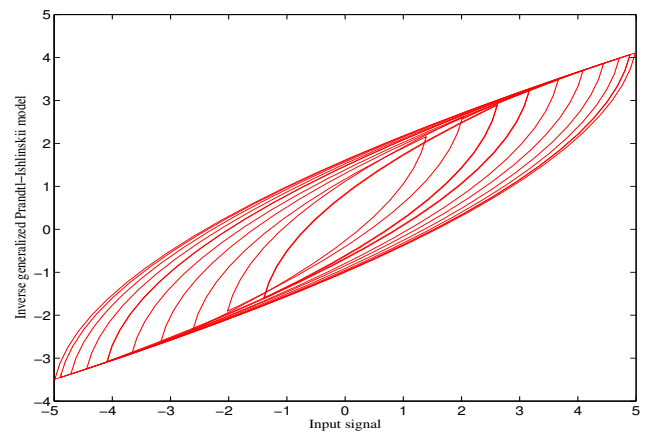

(b) Input-output relationship of the Inverse generalized PrandtlIshlinskii model.

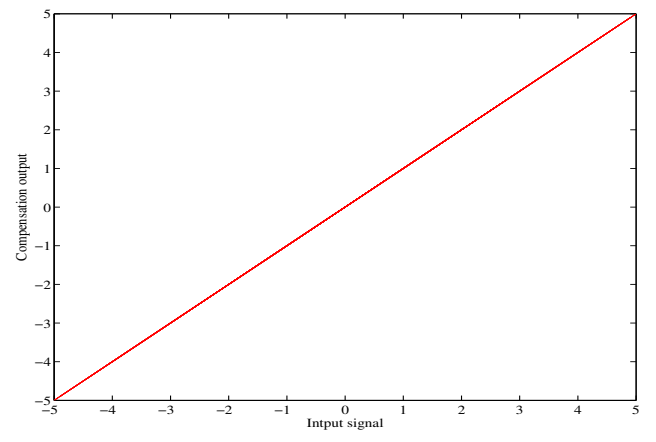

(c) Desired Output.

Fig. 3. Compensation hysteresis using Generalized Prandtl-Ishlinskii model and its inverse (Inverse feedforward compensator)

Consider the actual industrial system, it is reasonable to assume that

$$
w(t)=u(t)+d(t)
$$

where $d(t)$ is a bounded perturbation term to describe the disturbance, parametric uncertainties and measurement error etc. We use $D$ to denote its bound. Then, (19) can be reexpressed as

$$
\ddot{x}(t)+2 \xi \omega_{n} \dot{x}(t)+\omega_{n}^{2} x(t)=u(t)+d(t)
$$

The goal of the controller design is to guarantee the stability of the closed-loop system and optimize the tracking error in the presence of the linear dynamics. Focus on the system 
(24), the control objective is to design a control law for $u(t)$, to force the plant state $x(t)$ to follow a specified desired trajectory, $x_{d}(t)$, i.e., $x(t) \rightarrow x_{d}(t)$ as $t \rightarrow \infty$.

In the following development, we shall propose a robust adaptive controller for (24). Define

$$
x_{1}=x, x_{2}=\dot{x}
$$

then system (19) can be re-expressed as

$$
\begin{aligned}
\dot{x}_{1} & =x_{2} \\
\dot{x}_{2} & =u(t)-2 \xi \omega_{n} x_{2}(t)-\omega_{n}^{2} x_{1}(t)+d(t) \\
y & =x_{1}
\end{aligned}
$$

Define the tracking error $\tilde{x}$ as

$$
\tilde{x}=x-x_{d}
$$

and a filtered tracking error as

$$
s(t)=\left[\begin{array}{ll}
\lambda & 1
\end{array}\right]\left[\begin{array}{c}
\tilde{x}_{1} \\
\tilde{x}_{2}
\end{array}\right], \lambda>0
$$

In order to improve the precision, the tuning error $s_{\varepsilon}(t)$ is used,

$$
s_{\varepsilon}=s-\varepsilon \operatorname{sat}\left(\frac{s}{\varepsilon}\right)
$$

where $\varepsilon>0$ is a constant and $\operatorname{sat}(\cdot)$ is the saturation function, and the tuning error $s_{\varepsilon}$ disappears when $|s| \leq \varepsilon$. In order to present the developed adaptive control laws, the following definitions are required:

$$
\theta=\left[\begin{array}{l}
2 \xi \omega_{n} \\
\omega_{n}^{2}
\end{array}\right]
$$

then, we can define

$$
\begin{gathered}
\tilde{\theta}=\hat{\theta}-\theta \\
\tilde{D}=\hat{D}-D
\end{gathered}
$$

where $\hat{\theta}$ is the estimation of $\theta$ and $\hat{D}$ is the estimation of $D$ which is the boundedness of the $d(t)$. For the given plant, we propose the following control laws:

$$
u(t)=-k_{d} s-\lambda\left(x_{2}-\dot{x}_{d}\right)+\ddot{x}_{d}+\left[\begin{array}{l}
x_{1} \\
x_{2}
\end{array}\right]^{T} \hat{\theta}-\operatorname{sat}\left(\frac{s}{\varepsilon}\right) \hat{D}
$$

where $k_{d}$ and $\lambda$ are positive constants. The estimated parameters $\hat{\theta}$ and $\hat{D}$ will be updated by adaptive laws

$$
\begin{aligned}
& \dot{\hat{\theta}}=-\gamma \mathbf{x}^{T} s_{\varepsilon} \\
& \dot{\hat{D}}=\eta\left|s_{\varepsilon}\right|
\end{aligned}
$$

where $\gamma$ and $\eta$ are positive constants.

Theorem: For the system described by (26), with the hysteresis (21), and the inverse of the hysteresis (22), the control law given by (33) and the adaptive control law given by (34) ensure that all the closed-loop signals are bounded and the system vector $\mathbf{x}(t)$ converges to $\Omega_{\varepsilon}=\left\{\mathbf{x}(t)|| \tilde{x}_{i} \mid \leq\right.$ $\left.2^{i-1} \lambda^{i-2} \varepsilon, i=1,2\right\}$ for $\forall t \geq t_{0}$.
Proof: For system (26), the time derivative of the filtered error (28) is:

$$
\begin{aligned}
\dot{s}(t)= & \lambda\left(\dot{x}_{1}-\dot{x}_{d}\right)+\left(\dot{x}_{2}-\ddot{x}_{d}\right) \\
= & \lambda\left(\dot{x}_{1}-\dot{x}_{d}\right)+\left(u(t)-2 \xi \omega_{n} x_{2}-\omega_{n}^{2} x_{1}+d(t)\right) \\
& -\ddot{x}_{d}
\end{aligned}
$$

Applying the control law (33), we have

$$
\begin{aligned}
s_{\varepsilon} \dot{s}= & s_{\varepsilon}\left[-k_{d} s+\left[\begin{array}{l}
x_{1} \\
x_{2}
\end{array}\right]^{T} \hat{\theta}-2 \xi \omega_{n} x_{2}-\omega_{n}^{2} x_{1}\right. \\
& \left.-\operatorname{sat}\left(\frac{s}{\varepsilon}\right) \hat{D}+d(t)\right]
\end{aligned}
$$

To establish global boundedness, the following positive definite Lyapunov function candidate is adopted

$$
V=\frac{1}{2} s_{\varepsilon}^{2}+\frac{1}{\gamma}(\hat{\theta}-\theta)^{T}(\hat{\theta}-\theta)+\frac{1}{2 \eta} \tilde{D}^{2}
$$

Since the discontinuity at $|s|=\varepsilon$ is of the first kind and $s_{\varepsilon}=0$ when $|s| \leq \varepsilon$, it follows that the derivative $\dot{V}$ exists for all $s$, which is

$$
\dot{V}(t)=0, \text { when }|s| \leq \varepsilon
$$

When $|s|>\varepsilon$ and the fact that $s_{\varepsilon} \dot{s}_{\varepsilon}=s_{\varepsilon} \dot{s}$, we have

$$
\dot{V}=\frac{1}{2} s_{\varepsilon} \dot{s}+\frac{1}{\gamma}(\hat{\theta}-\theta)^{T} \dot{\hat{\theta}}+\frac{1}{\eta} \tilde{D} \dot{\tilde{D}}
$$

then, we can obtain

$$
\begin{aligned}
\dot{V}= & s_{\varepsilon} \dot{s}+\frac{1}{\gamma}(\hat{\theta}-\theta)^{T} \dot{\hat{\theta}}+\frac{1}{\eta} \tilde{D} \dot{\tilde{D}} \\
= & -k_{d} s_{\varepsilon} s+s_{\varepsilon}\left[\begin{array}{c}
x_{1} \\
x_{2}
\end{array}\right]^{T} \tilde{\theta}-s_{\varepsilon} \operatorname{sat}\left(\frac{s}{\varepsilon}\right) \hat{D}+s_{\varepsilon} d(t) \\
& +\frac{1}{\gamma}(\hat{\theta}-\theta)^{T} \dot{\hat{\theta}}+\frac{1}{\eta} \tilde{D} \dot{\tilde{D}} \\
\leq & -k_{d} s_{\varepsilon}^{2}+s_{\varepsilon}\left[\begin{array}{c}
x_{1} \\
x_{2}
\end{array}\right]^{T} \tilde{\theta}-\left|s_{\varepsilon}\right| \hat{D}+\left|s_{\varepsilon}\right| D \\
& +\frac{1}{\gamma}(\hat{\theta}-\theta)^{T} \dot{\hat{\theta}}+\frac{1}{\eta} \tilde{D} \dot{\tilde{D}}
\end{aligned}
$$

Utilizing the adaptive law given in (34), we can obtain

$$
\dot{V} \leq-k_{d} s_{\varepsilon}^{2}
$$

It can easily be shown that (35) is bounded, and $\dot{V}(t) \leq 0$ for all $t$, by Barbalat's lemma, we can prove that $\dot{V}(t) \rightarrow 0$, therefore, from (41), is can be shown that $s_{\varepsilon}(t) \rightarrow 0$ as $t \rightarrow \infty$, which indicates that $\tilde{\mathbf{x}}(t)$ will converge to $\Omega_{\varepsilon}$.

\section{B. Simulation Results}

In this simulation, for the hysteresis existing in the piezoceramic actuator, the same density function $p(r)=$ $0.1 e^{-0.1 r}, r \in[0,5]$ and the envelope function defined in (18) are chosen. The dynamics parameters $\zeta$ and $\omega_{n}$ are identified as 0.8 and $100 \mathrm{~Hz}$. According to the compensation scheme introduced in Section IV, we can obtain

$$
\begin{aligned}
\dot{x}_{1} & =x_{2} \\
\dot{x}_{2} & =u(t)-160 x_{2}(t)-10000 x_{1}(t)+d(t) \\
y & =x_{1}
\end{aligned}
$$


Utilizing the control law in (33) and the adaptive law in (34), the parameter values are chosen as $\gamma=10, k_{d}=2500$, $\lambda=200, \varepsilon=0.001$ and $\eta=0.001$. The initial state values are $x_{1}(0)=2, x_{2}(0)=7, \hat{\theta}(0)=[10,100]^{T}$ and $\hat{D}(0)=$ 0.01 , the desired trajectory $x_{d}$ is $3 \sin (0.75 \pi t)+2 \cos (0.5 \pi t)$. The tracking error is shown in Fig. 4, and the displacement response of the actuator system is shown in Fig. 5. We can see that the proposed controller clearly demonstrates excellent tracking performance and the control algorithm can overcome the effects of linear dynamics in the piezoceramic actuators.

\section{CONCLUSION}

Compensation of hysteresis nonlinearities is carried out using inverse generalized Prandtl-Ishlinskii model. Simulation results show the capability of the inverse generalized Prandtl-Ishlinskii model (inverse feedforward compensator) to compensate hysteresis nonlinearities. Considering the system dynamics and the system disturbance, a robust adaptive control scheme is developed to improve the robustness and ensure the stability of the closed-loop piezoceramic actuator system. Simulation results have confirmed the effectiveness of the proposed control approach.

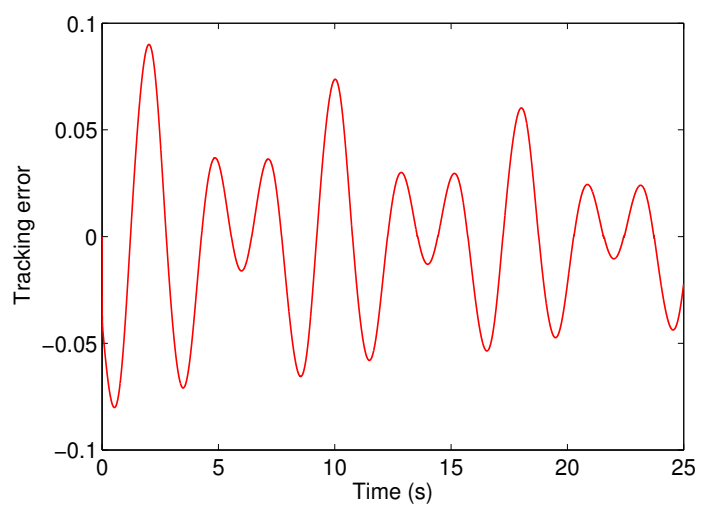

Fig. 4. Tracking error of the actuator system.

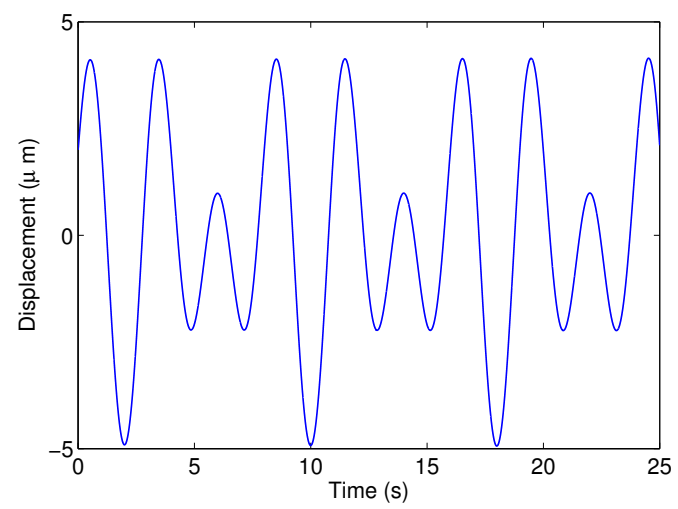

Fig. 5. The displacement response of the actuator system.

\section{REFERENCES}

[1] R.C. Smith, Smart Material System: Model Development, society for Industrial and Applied Mathematics, 2005.

[2] I.D. Mayergoyz, Mathematical Models of Hysteresis, New York, Elsevier, 2003.

[3] M. Brokate and J. Sprekels, Hysteresis and Phase Transitions, New York, Springer, 1996.

[4] M. Krasnoselskii and A. Pokrovskii, Systems with Hysteresis, Nauka, Moscow 1983; Springer-Verlarg Inc., 1989.

[5] G. Tao and P. V. Kokotovic, "Adaptive control of plant with unknown hysteresis", IEEE Transactions on Automatic Control, vol. 40, pp. 200212, 1995.

[6] P. Krejci and K. Kuhnen, "Inverse control of systems with hysteresis and creep," IEE Proc Control Theory Application, vol. 148, no.3, pp. 185-192, 2001.

[7] R. C. Smith, S. Seelecke, Z. Ounaies and J. Smith , "A Free Energy Model for Hysteresis in Ferroelectric Materials," Journal of Intelligent Materials Systems and Structures, vol. 14, no. 11, pp.719-739, 2003.

[8] R. B. Gorbet, D. Wang and K A Morris, "Preisach model identification of a two-wire SMA actuator," Proc. IEEE Int. Conf. on Robotics and Automation 3, pp. 2161-2168, 1998.

[9] D. Hughes and J. Wen, "Preisach modelling of piezoceramic and shape memory alloy hysteresis," Smart Materials and Structures, vol.6, pp.287-300, 1997.

[10] W. Galinaities, "Two methods for modeling scalar hysteresis and their use in controlling actuators with hysteresis," Ph.D. dissertation, Dept. Math., Blacksburg, Virginia, USA, 1999.

[11] G. Song, J. Zhao, X. Zhou, and J.A.D. Abreu-Garcia, "Tracking control of a piezoceramic actuator with hysteresis compensation using inverse Preisach model," IEEE Transactions on Mechatronics, vol. 10, no. 2, pp. 198-209, 2005.

[12] C. Y. Su, Q. Q. Wang, X. K. Chen, S. Rakheja, "Adaptive variable structure control for a class of nonlinear systems with unknown Prandtl-Ishlinskii hysteresis," IEEE Trans, Automat. Contr., vol. 50, no. 12, pp. 2069-2074, 2005.

[13] P. Ge and M. Jouaneh, "Modeling hysteresis in piezoceramic actuators," Precision Engineering, vol. 17, no. 3, pp. 211-221, 1995.

[14] H.T. Banks, A.J. Kurdila, and G. Webb, "Identification of hysteretic control influence operators representing smart actuators," Mathematical Problems in Engineering, vol. 3, no. 4, pp. 287-328, 1997.

[15] J. Nealis and R.C. Smith, "Model-Based Robust Control Design for Magnetostrictive Transducers Operating in Hysteretic and Nonlinear Regimes," IEEE Trans. on Control Systems Technology, vol. 15, no. 1, pp. 22-39, 2007.

[16] R.C. Smith and R. Zrostilk, "Inverse compensation for ferromagnetic hysteresis," Proc. IEEE Conference on Decision and Control, Phoenix, Arizona, pp. 2875-2880, 1999.

[17] X. Tan and H. Khalil, "Control Unknown Dynamic hysteretic systems using slow adaption: preliminary results," Proc. American Control Conference, NY, New York, pp. 3294-3299, 2007.

[18] P. Ge and M. Jouaneh, "Tracking control of a piezoceramic actuator," IEEE Trans. on Control Systems Technology, vol. 4, no. 3, pp. 209216, 1996.

[19] A. Visintin, Differential Models of Hysteresis, Springer Berlin, 1994.

[20] R. V. Iyer, X. Tan and P. S. Krishnaprasad , "Approximate inversion of the Preisach hysteresis operator with application to control of smart actuators," IEEE Trans. on Automatic Control, vol. 50, no. 6, pp. 798$810,2005$.

[21] X. Tan and J.S. Baras, "Modeling and Control of Hysteresis in Magne tostrictive Actuators," Automatica, vol. 40, no. 9. pp. 1469-1480, 2004.

[22] M. A. Janaideh, J. Mao, S. Rakheja, W. Xie, and C.-Y. Su, "Generalized Prandtl-Ishlinskii hysteresis model: Hysteresis modeling and its inverse for compensation in smart actuators," Proc. IEEE Conference on Decision and Control, Cancun, Mexico, pp. 5182-5187, 2008. 